"The final publication is available at Springer via $h t t p: / / d x . d o i . o r g / D O I$ 10.1007/s10404-014-1351-9" 


\title{
Rapid mold-free manufacturing of microfluidic devices with robust and spatially directed surface modifications
}

\author{
Gaspard Pardon - Farizah Saharil • J. \\ Mikael Karlsson • Omkar Supekar . \\ Carl Fredrik Carlborg . Wouter van der \\ Wijngaart • Tommy Haraldsson
}

Received: date / Accepted: date

\begin{abstract}
A new method that allows for mold-free, rapid and easy-to-use prototyping of microfluidic devices comprising channels, access holes and surface modified patterns, is presented. The innovative method is based on direct photolithographic patterning of an off-stoichiometry thiol-ene (OSTE) polymer formulation, tailor-made for photolithography, which offers unprecedented spatial resolution and allows for efficient, robust and reliable, room temperature surface modification and glue-free, covalent room temperature bonding. This mold-free process does not require cleanroom equipment and therefore allows for rapid, i.e. less than one hour, design-fabricate-test cycles, using a material suited for larger scale production. The excellent photolithographic properties of this new OSTE formulation allow for unprecedented, for thiol-ene polymer systems, resolution patterning in hundreds of micrometers thick layers, $200 \mu \mathrm{m}$ thick in this work. Moreover, we demonstrated robust (covalent) and spatially-controlled modification of the microchannel surfaces with a contact angle of 76 degrees to hydrophobic/hydrophilic areas with contact angles of 102 and 43 degrees, respectively.
\end{abstract}

Keywords lab on chip · microfluidics · photolithography · OSTE polymer · surface modification

\section{Introduction}

For faster development of microfluidic applications, researchers need access to quick and easy manufacturing of microfluidic devices to verify designs and to

G. Pardon · F. Saharil · J. M. Karlsson · C. F. Carlborg · W. van der Wijngaart (凶) · T. Haraldsson

Micro and Nanosystems, KTH Royal Institute of Technology, Osquldas vg 10, SE-10044 Stockholm, Sweden

Tel.: +4687906613

Fax: +468100858

E-mail: wouter@kth.se

O. Supekar

Indian Institute of Technology Bombay, Powai, Mumbai, Maharashtra, 400076 (India) 
test their applications. In addition, for smooth transition to mass manufacturing, prototype devices must be fabricated with appropriate geometries and surface properties, in a repeatable manner, preferably using materials and methods with a realistic potential for larger scale production. [1,2] In this paper, we present the first comprehensive method for the rapid fabrication of fully functional microfluidic chips using only photopatterning.

Two types of techniques are available for microfluidic device manufacturing: those based on molding and those that are mold-free.[1,3] Each technique utilizes specific materials and associated back-end processing. Mold-based techniques include casting, imprinting, hot embossing and injection molding, and are well suited for mass-production but are generally too slow, cumbersome or expensive for efficient rapid manufacturing in a research setting. [4] Mold-free techniques, including lamination, [5,6] photolithography[7-9] and stereolithography, $[10,11]$ are attractive for low-volume manufacturing due to low cost and manufacturing flexibility. Some mold-free techniques can potentially be used for large-scale production using, for example, large-area patterning or roll-to-roll techniques. [12-14]

Moreover, the choice of the material and machining technique necessitates a related set of back-end processes, where the most important ones are surface modification and sealing of microfluidic channels. The latter is a well-known problem area where lamination, adhesive bonding or ultrasonic welding often are the only solutions. Surface modifications and their complexity and stability differ greatly between materials.[15-18] Hence, protocols suited for small-scale manufacturing are seldom transferrable to larger-scale production, in particular because the available materials differ between small and large-scale productions. In the case of moldfree techniques, surface modification has been reported for SU8, using plasma treatment activation[19,20] or chemical ring-opening of the residual epoxy groups together with amine or silane-based chemistry.[21-23] These methods suffer from either short shelf life or long multistep processing. As an alternative, the acrylatebased CLiPP process was proposed as an attempt to solve some of the limitations listed above. [24,25] However, this process faced limits in feature resolution and surface property control due to the difficult removal of the sacrificial wax material.

Overall, there exist no versatile hybrid solution, with a realistic potential for low and large-scale production using a single material with easy, reliable, longlasting back-end processing capabilities. Researchers need a technology bearing the hallmarks of soft-lithography, e.g. simple device layer fabrication and inexpensive tooling, while simultaneously allowing for prototyping of thermoplastic-like devices that can easily be converted into a mass-product form. Following this idea, the off-stoichiometry thiol-ene (OSTE) polymer system has been recently developed and has shown promising features, such as robust covalent bonding to various substrates, surface modification, and easy casting of the liquid prepolymer in a mold.[26] However, the mold preparation process, which is costly and slow, remains an unresolved issue for low volume, fast turn-around manufacturing.

Recently, a bonding technique for thiol-ene polymer [27] and a protein surface patterning method [28] were proposed. High resolution photolithography of a thiolene polymer was reported and, in separate contributions, surface modification by photographting of OSTE was introduced by our group at the MicroTAS conference 2012.[29,30] 
In this paper, we propose the first comprehensive method for the rapid fabrication of fully functional OSTE microfluidic chips using only photopatterning by building upon the recent advances in direct lithography and spatially directed surface modifications using a new OSTE formulation. This technology allows, for the first time, mold-free, rapid and easy-to-use prototyping of microfluidic devices comprising channels, access holes and robust surface modified patterns. The main techniques are: a) direct photolithographic patterning of an OSTE polymer formulation, tailor-made for photolithography, which offers unprecedented spatial resolution; b) efficient, robust and reliable, spatially directed, room temperature surface modification; and c) glue free, covalent room temperature bonding. This mold-free process does not require cleanroom equipment and therefore allows for rapid, i.e. less than one hour, design-fabricate-test cycles. The off-stoichiometric ratio of the monomers functional groups allows for robust covalent surface modifications via UV photografting and for reliable bonding of the sealing layer via click chemistry. We further demonstrate the spatially-controlled modification of an OSTE surface with a initial contact angle of 76 degrees to hydrophobic/hydrophilic areas with contact angles of 102 and 43 degrees, respectively.

In comparison with other microfluidic prototyping methods in PDMS, SU8 or thermoplastics, this process offers less complicated, more rapid and more robust (covalent) surface modifications and bonding. The whole process is performed at room temperature and does not require plasma treatment, adhesives, or molding. The fabrication of a demonstrator microfluidic device exhibiting very good pattern fidelity, excellent transparency and hydrophilic/hydrophobic surface patterns is shown.

\section{Materials and Methods}

\subsection{Materials selection}

OSTE is a polymer system, in which thiol and ene monomers are mixed in an off-stoichiometric ratio, and cured by a UV initiated "Click" polymerization. The choice of the thiol and ene (here, allyl) monomer, in addition to their mixing ratio, allows for design of specific polymer end-properties, e.g. Young modulus, $E_{g}$, and glass transition temperature $T_{g}$. As a first step, several monomer formulations have been evaluated with respect to the photolithographic properties, in particular the ease of solvent development of photolithographically defined microstructures, i.e. the removal of uncured monomer mixture after exposure. Indeed, due to the low solubility of oligomers, which are formed as intermediate polymerization products and which diffuse into the non-exposed regions, the development can be difficult in channels and high aspect ratio structures. We assume that the solubility of the oligomers is related to that of the monomers they are composed of. To minimize issues with insoluble oligomers, a qualitative solubility test was performed to find the most effective solvent for the available monomers. The best formulation is then used to fabricate a microfluidic chip and to characterize the manufacturing process. Several monomers were tested for solubility in selected solvents. The monomers tested were: Tetraallyloxyethane (TAOE) (Tokyo Chemical Industry Co., Ltd., Japan), Tris[2-(3-mercaptopropionyloxy) ethyl] Isocyanurate (TMPEIC) (Wako Chemical Inc., USA), 1,3,5-Triallyl-1,3,5-triazine-2,4,6(1H,3H,5H)-trione 
(TATATO) and Pentaerythritol tetrakis(2-mercaptoacetate) (PTMA) (Sigma-Aldrich Co., USA). Solvents used were isopropyl alcohol (IPA), acetone (SunChem AB, Sweden), butyl acetate (BuAc) and toluene (Merck KGaA, Germany). The monomers were dissolved in each solvent at a ratio of $20 \%$ wt. Monomers with low solubility form a precipitate, which is impermissible in the current application. The results, presented in Table 1, show that the best solvents are acetone and BuAc. However, the OSTE formulations used in this study swell slightly in acetone, which was therefore discarded. PTMA was dismissed because of its slow solubility in most solvents and TAOE was preferred to TATATO as allyl functional monomer, since TATATO was observed to homopolymerize, resulting in a less transparent material and likely more difficult to develop after exposure. Based on these findings, TAOE and TMPEIC were chosen for the OSTE formulation, with BuAC as the developer.

Two OSTE prepolymers were formulated: one with stoichiometry ratios of $40 \%$ thiol group excess (OSTE-Thiol 40) and one with $20 \%$ allyl group excess (OSTE-Allyl 20). These stoichiometry ratios are equivalent to a mix of $77.8 \% \mathrm{wt}$ TMPEIC and $20.2 \%$ wt TAOE; and $67.4 \%$ wt TMPEIC and $30.6 \%$ wt TAOE, respectively. $0.3 \%$ wt of Lucirin TPO-L photoinitiator (BASF Corp., USA); a bonding adhesion promoter (Mercene Labs AB, Sweden); and $1 \%$ wt of inhibitor Q1301 (Wako Chemical Inc., USA) in toluene were added, whose functionalities are discussed below. The OSTE was photopolymerized using a $12 \mathrm{~mW} \mathrm{~cm}^{-2}$ nearUV mercury lamp (OAI, Milpitas, USA).

\subsection{Fabrication process}

The chip fabrication, presented in Figure 1, consists of the following steps: A: Glass slides were silanized with the methacrylate functionalized Z-6030 Silane (Dow Corning Co., USA), mixed at $4 \% \mathrm{wt}$ in methanol, for $10 \mathrm{~min}$, followed by rinsing with IPA and a 10 min silanization reaction in a $110^{\circ} \mathrm{C}$ oven. This step enables covalent bonding between the silane tethered methacrylate and the subsequently applied OSTE layer, which facilitates further processing but is not required if the bottom layer is made thicker for a better handling capability. The bottom layer was prepared using OSTE-Thiol 40, which was photopolymerized for $3 \mathrm{~s}$. To ensure a layer with an even thickness, the OSTE-Thiol 40 was sandwiched between the glass slide and a flexible polyethylene release liner separated by $200 \mu \mathrm{m}$ spacers. A glass slide was used on top to obtain an even pressure distribution on the liner and ensure creating a flat layer. B: The middle layer, which defines the microchannels, was prepared following a similar procedure but OSTE-Allyl 20 was used instead. The release liner was here replaced by a high quality transparent photomask (Micro Lithography Services Ltd, UK) containing the microchannel design. The OSTE-Allyl 20 was exposed for $36 \mathrm{~s}$, in $3 \times 12$ $\mathrm{s}$ to avoid overheating, through a $400 \mathrm{~nm}$ cut-off glass filter (from Schott AG, Germany) to prevent consumption of the bonding adhesion promoter, which is activated at wavelengths below $370 \mathrm{~nm}$. The OSTE-Allyl 20 was developed at room temperature for $1 \mathrm{~min}$ in $\mathrm{BuAC}$ under constant agitation. After development, the chip was rinsed with IPA and dried with nitrogen gas. C: The top layer was prepared on a different substrate using OSTE-Thiol 40. Similarly to B, OSTE-Thiol 40 was sandwiched between a photomask and the substrate, using 
$200 \mu \mathrm{m}$ spacers to control the thickness. Here, a photomask with access-hole patterns was used. $2 \times 2=4 \mathrm{~s}$ of exposure without filter followed. The same development described in B was performed. D: The top layer was aligned manually onto the bottom layers, while carefully avoiding creation of air pockets. Finally, the bond was UV-activated with an unfiltered exposure of $20 \mathrm{~s}$. E: The microchannels were then surface modified. First, one region of the microchannel was made hydrophobic. The channels were filled with 3,3,4,4,5,5,6,6,7,7,8,8,9,9,10,10,- heptadecafluorodecylmethacrylate (FDM) (Sigma-Aldrich Co., USA) $20 \%$ wt in toluene, 0.1 \%wt Lucirin TPO-L and $0.1 \%$ wt benzophenone (BP). A photomask was placed on top of the chip to only expose the area to be modified (see Fig. 1.B., frame 1). The surface modification reaction was then activated with an unfiltered UV exposure of $90 \mathrm{~s}$, followed by careful rinsing with toluene for $1 \mathrm{~min}$ to remove nonspecifically bound molecules. The rest of the microchannel was made hydrophilic by repeating the procedure above, but using 2-hydroxyethyl methacrylate (HEMA) (Sigma-Aldrich Co., USA) $5 \%$ wt in toluene, $0.1 \%$ wt Lucirin TPO-L and $0.1 \%$ wt BP. For this step, a photomask with reverse polarity, dark field image, was used.

\section{Results and discussion}

First, the role of each component in the OSTE mixture is discussed. The TAOE and TMPEIC monomers carry four allyl and three thiol functional groups, respectively, whose total ratio was chosen to obtain two OSTE formulations with, respectively, $40 \%$ excess of the thiol groups and $20 \%$ excess of the allyl groups. The off-stoichiometric ratio makes thiol and, respectively, allyl groups available at the surface for further reaction, here bonding and surface modification. The TPO-L photoinitiator serves to initiate the cross-linking of the monomers upon UV radiation; the bonding adhesion promoter serves to activate the thiol groups, readily available at the surface through the off-stoichiometric thiol-ene mixture; and the inhibitor serves to increase the shelf-life of the monomer mix and to decrease the sensitivity of the OSTE to stray light during photolithography. The latter improves the photolithography development by reducing the amount of oligomers formed in the monomer mixture and in the non-exposed region, thus maintaining the viscosity to a level as low as possible. The bonding adhesion promoter is activated at wavelengths lower then $370 \mathrm{~nm}$, which can be preserved, by using a cut-off filter during the photolithography, for subsequent activation for bonding of layers together. No deep-UV is required to break surface bonds, since thiol or allyl groups are made readily available for reacting via the off-stoichiometric mixture of the thiol-ene functional groups. The compatibility of this promoter with useful optical sensing methods is discussed below.

Characterization of the chip after photolithography was made using optical and SEM microscopy. To decrease electrostatic charging during imaging, $30 \mathrm{~nm}$ of gold was evaporated onto the structures. Figure 2.A. shows SEM images of the microchannels before the lid was bonded to seal the chip. The microchannels were $400 \mu \mathrm{m}$ wide and $200 \mu \mathrm{m}$ high. The pattern fidelity was excellent despite the $200 \mu \mathrm{m}$ layer thickness. The vertical lines, noticed on the close-up images, likely resulted from the diffraction of light by microscopic air bubbles trapped between the mask and the OSTE polymer. A slight positive tapering of the channel wall was a consequence of a slight overexposure. However, the presence of this tapering 
indicates that very little broadening of the features occurred, which is in stark contrast with the broadening in stoichiometric thiol-ene reported by Bowman et al. [31] Our group has recently reported an aspect ratio of 1:8 for photopatterned micropillars [29]. For the OSTE formulation used in this work, aspect ratio tests have been performed and revealed that an aspect ratio 1:4 were achievable without difficulties for both pillar and hole structures. For these tests, we fabricated 100 $\mu \mathrm{m}$ circular pillars and holes in $400 \mu \mathrm{m}$ thick OSTE layers. For higher aspect ratio, pillars tend to collapse during development and the development became difficult for holes, although optimization still remains possible. The process was performed on the chip level but could easily be transposed to larger area processing. Finally, the images of Figure 2.A show the excellent surface quality of the cured polymer, where the polymer surface remained undamaged by the removal of the release layer/photomask from the cured polymer.

Contact angle measurements were performed on flat OSTE samples that were modified using the protocol described under point E. The measurements of the hydrophobic and hydrophilic surface modifications were performed on several substrates each using a minimum of 6 independently measured $5 \mu \mathrm{L}$ droplets per substrate. The results are reported in Figure 3. The surface was modified from a native surface with contact angle of 76 degrees to hydrophobic/hydrophilic areas with contact angles of 102 and 43 degrees, respectively, leading to 59 degrees difference in contact angle between the hydrophobic/hydrophilic regions.

Microscope images of the final microfluidic chip is shown in Figure 2.B. The design chosen for demonstration consisted of one inlet on the left, leading to a chamber followed by a meander. The channel then splits in two branches leading to two outlets. The superimposed dashed line shows the position of the mask during hydrophobic and hydrophilic surface modification. Of the two channels on the right side, one was made hydrophobic to prevent the liquid to reach that outlet, as can be seen on the filmstrip images, while the rest of the channel was made hydrophilic. Figure 2.C. shows the optical clarity of OSTE, as the institution logo is clearly visible through our $600 \mu \mathrm{m}$ thick device.

The optical properties of the material were assessed in terms of autofluorescence at several emission wavelengths of relevance for bio-imaging, as reported in Figure 4. The autofluorescence was measured relatively to that of borosilicate glass at the same wavelength after $180 \mathrm{~s}$ illumination. The insert shows the autofluorescence over time at emission wavelength, $\lambda_{e m}=445 \mathrm{~nm}$, where a maximum is reached after $180 \mathrm{~s}$ before photobleaching occurs. The data compare well with values reported for other relevant material such as COC and PMMA.[32]

The bonding quality was investigated using fluorescence imaging with Rhodamine B, as shown in Figure 4. The chip was incubated for $69 \mathrm{~h}$ and neither creeping of the fluorescent dye at the bond interface, nor diffusion of the dye inside the polymer could be observed. The bonding quality of the lid was further assessed by performing a blister test on a fabricated chip to evaluate the maximum pressure it can withstand. The two outlets of the chip were plugged while the inlet was connected to a pressure sensor and to pressure regulated nitrogen gas, using a tailor-made gasket and holder. The chip could withstand up to 4.13 bar before delamination of the layers began to occur, followed by rupturing of the chip lid. Higher pressures are only required for specific and demanding microfluidic applications, making the proposed technique widely useful. 
Table 1 Solubility test of several thiol and allyl functional monomer in various solvents. A denotes that the monomer has very limited solubility in the corresponding solvent, $a+$ that it dissolves when approaching $20 \% \mathrm{wt}$ under strong agitation and a ++ that it dissolve without only little agitation and that no precipitate is observed at $20 \%$ wt.

\begin{tabular}{lllll}
\hline & IPA & Acetone & BuAc & Toluene \\
\hline TAOE & + & ++ & ++ & + \\
TMPEIC & - & ++ & ++ & + \\
TATATO & + & ++ & ++ & + \\
PTMA & - & + & + & - \\
\hline
\end{tabular}

The fabrication process detailed in Scheme 1 consists of only five major steps for the fabrication of a complete microfluidic chip, including surface modifications and microfluidic connection ports. We performed the entire fabrication, excluding the mixing of the monomers, in less than one hour without the need for clean room processing equipment. This makes this technique interesting for lab-on-chip application-focused laboratories and clearly superior to PDMS-based rapid prototyping or SU8 based methods in terms of ease of manufacturing and performance, especially with respect to the potential control and stability of the surface modifications. The fabricated chips are directly usable for testing various applications. The surface modification, demonstrated here, allows for precise surface energy control, and other chemical groups could be grafted onto the surface in a similar way, e.g. for biofunctionalization.

\section{Conclusion}

The fabrication method presented in this work provides a number of innovations. The direct lithography of OSTE polymer enables the fabrication of microfluidic chips without requiring any mold. The microfluidic features, channel and access holes, are directly defined in the polymer by photolithography. The optimized choice of the monomers and solvent, with respect to the photolithography development, results in an excellent resolution of the photolithography, even in relatively thick layers. The OSTE polymer utilizes thiol-ene click chemistry for unassisted, efficient and reliable bonding of the top lid and for spatially directed surface modifications. The fabricated chip is made entirely from the same OSTE material, differing only in off-stoichiometric ratio, demonstrating the versatility of the OSTE concept where new materials are easily developed by simple adjustments of monomer concentrations. The microchannel surface energy is locally modified in a robust fashion by photolithographically controlled grafting. The material shows excellent transparency, which is important for many detection schemes.

The fabrication method is uncomplicated and rapid, making it method attractive for a large range of laboratory settings. Finally, the proposed solution has a potential for larger scale manufacturing by increasing the surface area. This was, until now, an unresolved issue with other techniques and materials.

\section{References}

1. C.D. Chin, V. Linder, S.K. Sia, Lab On A Chip 12(12), 2118 (2012) 


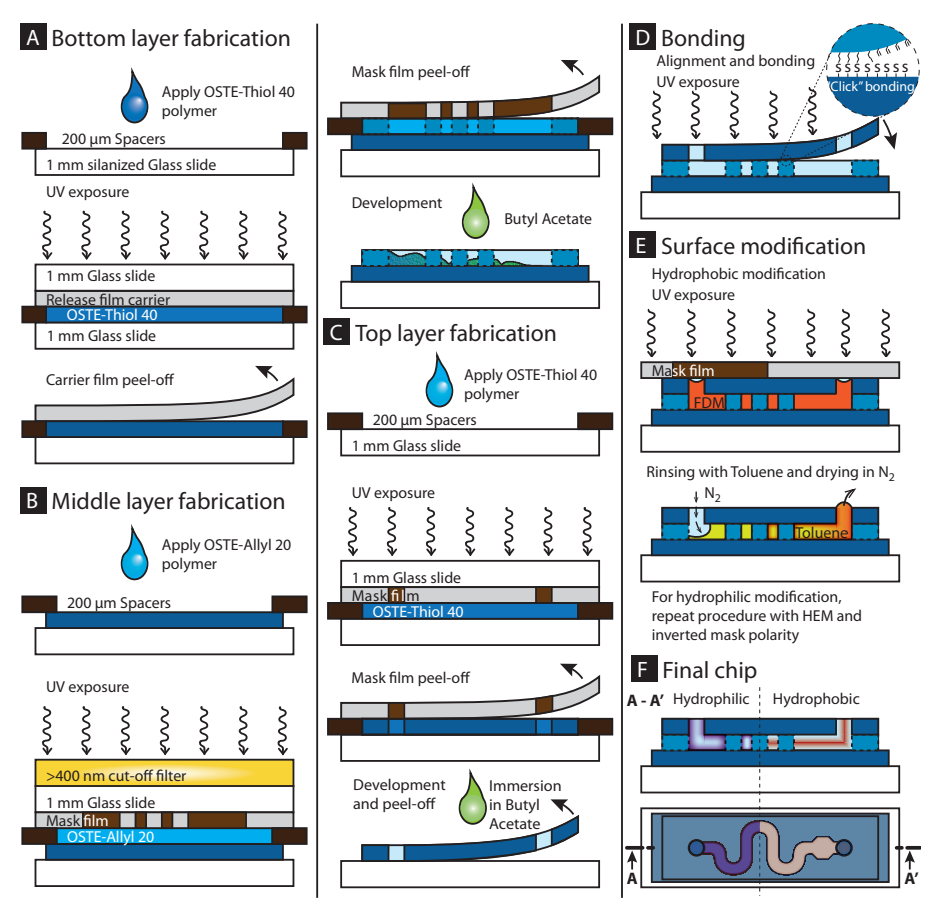

Fig. 1 Process flow for the fabrication of surface modified microfluidic chips using direct lithography on OSTE polymer. A: Fabrication of the bottom layer by UV curing the OSTE polymer. B: Fabrication of the middle layer, where microchannels are defined by photolithography of OSTE polymer. C: Fabrication of the top layer, where the access holes are defined by photolithography of OSTE polymer. D: UV activated bonding of the top layer on the middle layer to seal the microfluidic channels. E: UV activated, spatially directed surface modification, performed once for hydrophobic and once for hydrophilic modification. F: Final microfluidic chip with patterned surface energy.

2. R. Tantra, H. van Heeren, Lab On A Chip 13(12), 2199 (2013)

3. H. Becker, C. Gärtner, Analytical and bioanalytical chemistry 390(1), 89 (2007)

4. O. Rötting, W. Röpke, H. Becker, C. Gärtner, Microsystem Technologies 8(1), 32 (2002)

5. D.A. Bartholomeusz, R.W. Boutte, J.D. Andrade, Microelectromechanical Systems, Journal of 14(6), 1364 (2005)

6. P.K. Yuen, V.N. Goral, Lab On A Chip 10(3), 384 (2010)

7. H. Sato, H. Matsumura, S. Keino, S. Shoji, Journal of Micromechanics and Microengineering 16(11), 2318 (2006)

8. B. Bohl, R. Steger, R. Zengerle, P. Koltay, Journal of Micromechanics and Microengineering 15(6), 1125 (2005)

9. J. Zhang, M.B. Chan-Park, S.R. Conner, Lab On A Chip 4(6), 646 (2004)

10. K.S. Lee, R.H. Kim, D.Y. Yang, S.H. Park, Progress in Polymer Science 33(6), 631 (2008)

11. F.P.W. Melchels, J. Feijen, D.W. Grijpma, Biomaterials 31(24), 6121 (2010)

12. R. Liedert, L.K. Amundsen, A. Hokkanen, M. Mäki, A. Aittakorpi, M. Pakanen, J.R. Scherer, R.A. Mathies, M. Kurkinen, S. Uusitalo, L. Hakalahti, T.K. Nevanen, H. Siitari, H. Söderlund, Lab On A Chip 12(2), 333 (2012)

13. S.H. Ng, Z.F. Wang, Microsystem Technologies 15(8), 1149 (2008)

14. L.P. Yeo, S.H. Ng, Z. Wang, Z. Wang, N.F. de Rooij, Microelectronic Engineering 86(4-6), 933 (2009)

15. L.E. Locascio, A.C. Henry, T.J. Johnson, D. Ross, Lab On A Chip (2003)

16. G.A. Diaz-Quijada, R. Peytavi, A. Nantel, E. Roy, M.G. Bergeron, M.M. Dumoulin, T. Veres, Lab On A Chip 7(7), 856 (2007)

17. D. Belder, M. Ludwig, Electrophoresis 24(21), 3595 (2003) 


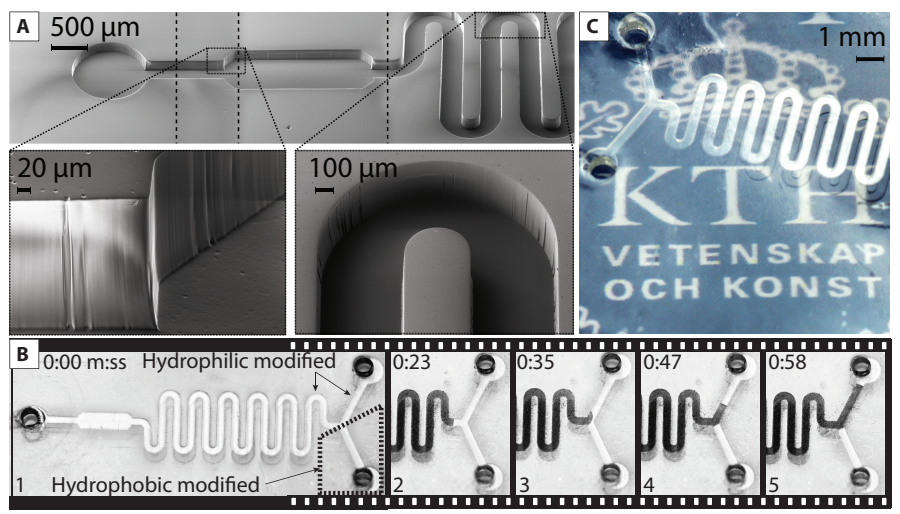

Fig. 2 A. SEM images of the microchannel defined using lithography. Several images were stitched together and the stitch lines are shown on the images. Two close-ups are also shown at the bottom. B. Picture of a microchannel extending in a filmstrip showing the capillary filling of the chip with a dye solution. On frame 3, the capillary filling stops when reaching the hydrophobic-modified region and only fills the uppermost hydrophilic channel. The position of the mask used for hydrophobic modification is shown with dashed lines and the time lapse for each frame is mentioned. (Image contrast was increased for better visibility) C. Photography of a complete microfluidic chip showing in transparency our institution logo. This demonstrates the very high transparency of the polymer.

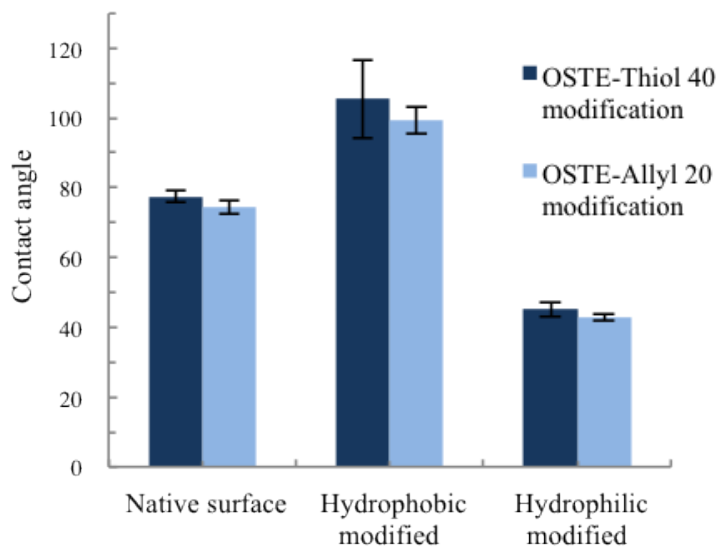

Fig. 3 Contact angle measurements of the OSTE surfaces before and after surface hydrophobic/philic modifications. The error bar shows the standard deviation on minimum 6 measurements.

18. J. Zhou, D.A. Khodakov, A.V. Ellis, N.H. Voelcker, ELECTROPHORESIS 33(1), 89 (2012)

19. F. Walther, P. Davydovskaya, S. Zuecher, M. Kaiser, H. Herberg, A.M. Gigler, R.W. Stark, Journal of Micromechanics and Microengineering 17(3), 524 (2007)

20. F. Walther, T. Drobek, A.M. Gigler, M. Hennemeyer, M. Kaiser, H. Herberg, T. Shimitsu, G.E. Morfill, R.W. Stark, Surface and Interface Analysis 42(12-13), 1735 (2010)

21. M. Joshi, R. Pinto, V.R. Rao, S. Mukherji, Applied Surface Science 253(6), 3127 (2007)

22. K. Qvortrup, K.M. Taveras, O. Thastrup, T.E. Nielsen, Chemical Communications 47(4), 1309 (2011)

23. Y. Wang, J.H. Pai, H.H. Lai, C.E. Sims, M. Bachman, G.P. Li, N.L. Allbritton, Journal of Micromechanics and Microengineering 17(7), 1371 (2007) 

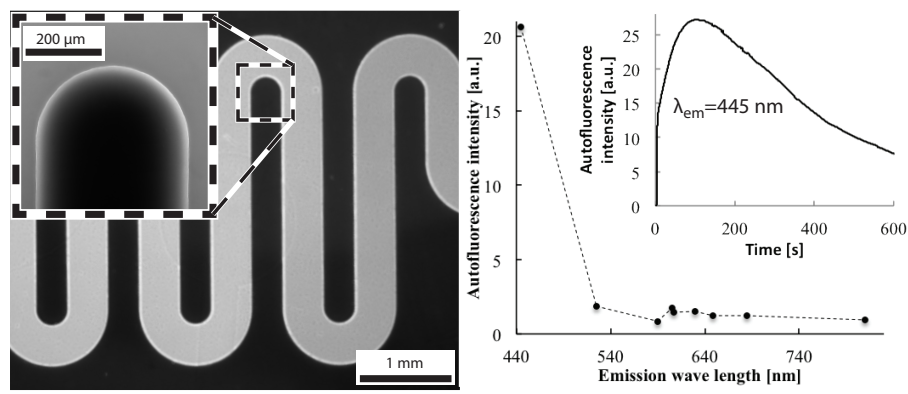

Fig. 4 Left: Fluorescence images of the microchannels filled with Rhodamine B solution, 69 $\mathrm{h}$ after injection. No leakage could be detected indicating a leak free bond interface. Right: Measurements of the autofluorescence of the OSTE at several emission wavelengths of relevance for bio-imaging. The insert shows the autofluorescence at $445 \mathrm{~nm}$ emission wavelength over time, indicating that photobleaching occurs.

24. J.B. Hutchison, K.T. Haraldsson, B.T. Good, R.P. Sebra, N. Luo, K.S. Anseth, C.N. Bowman, Lab On A Chip 4(6), 658 (2004)

25. T. Haraldsson, Hutchison, Sebra, Good, Anseth, Bowman, Sensors And Actuators BChemical 113(1), 7 (2006)

26. C.F. Carlborg, T. Haraldsson, K. Öberg, M. Malkoch, W. van der Wijngaart, Lab On A Chip 11(18), 3136 (2011)

27. T.M. Sikanen, J.P. Lafleur, M.E. Moilanen, G. Zhuang, T.G. Jensen, J.P. Kutter, Journal of Micromechanics and Microengineering 23(3), 037002 (2013)

28. J.P. Lafleur, R. Kwapiszewski, T.G. Jensen, J.P. Kutter, Analyst 138(3), 845 (2013)

29. J.M. Karlsson, C.F. Carlborg, F. Saharil, F. Forsberg, F. Niklaus, W. van der Wijngaart, T. Haraldsson, in 16th International Conference on Miniaturized Systems for Chemistry and Life Sciences (2012), pp. 225-227

30. C.F. Carlborg, F. Moraga, F. Saharil, W. van der Wijngaart, T. Haraldsson, in 16th International Conference on Miniaturized Systems for Chemistry and Life Sciences (2012), pp. $677-679$

31. C.N. Bowman, C.J. Kloxin, AIChE Journal 54(11), 2775 (2008)

32. A. Piruska, I. Nikcevic, S.H. Lee, C. Ahn, W.R. Heineman, P.A. Limbach, C.J. Seliskar, Lab On A Chip 5(12), 1348 (2005) 